\title{
Fragen zum Thema „Chronische Pankreatitis“
}

\section{- 1. Eine fehlende Amylase- bzw. Lipaseerhöhung bei Patienten mit akuten Oberbauchbeschwer- den}

A ist immer ein Hinweis auf ein Gallensteinleiden.

B ist immer ein Hinweis auf ein peptisches Ulkus.

C ist immer ein Hinweis auf ein Kolonkarzinom.

D ist immer ein Hinweis auf ein Aortenaneurysma.

E schließt eine chronische Pankreatitis nicht aus.

\section{-2. Normale Stuhlenzymwerte (Chymotrypsin,} Elastase)

A schließen eine exokrine Pankreasinsuffizienz aus.

B schließen eine exokrine Pankreasinsuffizienz nicht aus.

C beweisen ein irritables Magen-Darm-Syndrom.

D sind immer ein Hinweis auf ein Ulcus duodeni.

E sind immer ein Hinweis auf eine akute Pankreatitis.

\section{- 3. Ein Haemosuccus pancreaticus}

A ist ein Speichelödem der Bauchspeicheldrüse.

B ist eine Absaugvorrichtung für den Pankreassaft.

C ist eine Blutung in den Pankreasgang.

D ist eine Blutung aus einem papillennahen Ulcus duodeni.

E ist eine intraperitoneale Blutung.
-4. Der Erfolg einer Enzymtherapie kann kontrolliert werden

A durch Messung des Körpergewichts und/ oder durch Stuhlgewicht- und Stuhlfettanalyse.

B durch Messung des Hämatokrits und des Gesamteiweißes.

C durch Beurteilung des Hautturgors.

D durch Kontrolle auf Nachtblindheit (Vita$\min$ A).

E durch Überprüfung der Schmerzsymptomatik.

\section{-5. Eine chirurgische Therapie bei chronischer} Pankreatitis sollte durchgeführt werden

A bei nicht beherrschbaren Schmerzen.

B bei Ulcus duodeni, bedingt durch das saure Milieu im Duodenum bei exokriner Pankreasinsuffizienz.

C bei Gewichtsabnahme.

D bei Diabetes mellitus.

E bei zunehmender exokriner Pankreasinsuffizienz.

\section{-6. Der sensitivste Test zum Beweis einer exokri-} nen Pankreasinsuffizienz ist

A der orale Glukosetoleranztest.

B der Sekretin-Caerulein-Test.

C die Chymotrypsinbestimmung im Stuhl.

D die Elastase-1-Bestimmung.

E der Pankreolauryltest.

\section{- 7. Bei nachgewiesener exokriner Pankreas-} insuffizienz sollte

A der Patient nicht mehr als 1400 kcal pro Tag zu sich nehmen.

B unbesehen mit Enzymen substituiert werden.
C eine proteinarme Diät verordnet werden

D das Stuhlgewicht und die Stuhlfettausscheidung über 72 Stunden bestimmt werden.

E eine fettreiche Diät verordnet werden.

- 8. Welcher Befund spricht am ehesten für die Diagnose einer chronischen Pankreatitis?

A Oberbauchschmerzen.

B Wässrige Durchfälle.

C Verkalkungen in der CT.

D Pankreasgangveränderungen in der ERCP.

E Steatorrhö.

9. Der sekundäre Diabetes mellitus von Patienten mit endo- und exokriner Pankreasinsuffizienz zeichnet sich durch welche Besonderheit aus?

A Hoher Insulinbedarf wegen Insulinresistenz.

B Geringer Insulinbedarf und Hypoglykämieneigung.

C Überschießende Glukagonsekretion.

D Neigung zur Ketoazidose.

E Antikörperbildung gegen Humaninsulin.

- 10. Welche zusätzliche Therapie zur Enzymsubstitution benötigt ein Patient mit chronischer Pankreasinsuffizienz?

A Statine zur Cholesterinsenkung.

B Vitamin C zur Prophylaxe von Skorbut.

C Fettlösliche Vitamine A, D, E, K parenteral.

D Schilddrüsenhormone.

E Ursodesoxycholsäure.

\section{ANTWORTFORMULAR}

Ich versichere, alle Fragen ohne fremde Hilfe beantwortet zu haben.

Mit dem Einreichen dieses Fragebogens erklärt sich der Einreichende damit einverstanden, dass die angegebenen Daten zum Zweck der Teilnahmebestätigung gespeichert und bei erfolgreicher Teilnahme auch an den Einheitlichen Informationsverteiler (EIV) der Ärztekammern weitergegeben werden.
„Chronische Pankreatitis“

MMW-Fortschr. Med. Nr. 45 / 2006 (148. Jg.)

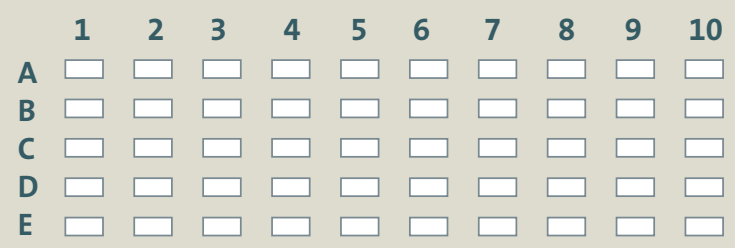

Bitte kreuzen Sie pro Frage je eine Antwort deutlich an.
Und so kommen Sie zu Ihren Punkten:

Teilnahme im Internet: unter www.cme-punkt.de. Dort führen wir für Sie ein elektronisches Punktekonto.

Teilnahme per Brief Fragebogen ausfüllen und mit einem frankierten Rückumschlag an: Urban \& Vogel Medien und Medizin Verlagsgesellschaft, Redaktion MMW-Fortschritte der Medizin, Abt. Fortbildungszertifikat, Neumarkter Str. 43, 81673 München. 